\title{
Uncatalyzed Friedel-Crafts Alkylation of Aromatic Compounds Through Reactive Benzyl Cations Generated From $N$-Sulfamoylcarbamates
}

\author{
Michael Sefkow, ${ }^{*}$ Jens Buchs \\ Universität Potsdam, Institut für Chemie, Karl-Liebknecht- \\ Strasse 24-25, D-14476 Golm, Germany. \\ sefkow@rz.uni-potsdam.de
}

Supporting Information.

\section{Genreral Experimental}

All reactions were performed in dried glassware under an inert $\left(\mathrm{N}_{2}\right)$ atmosphere. Standard reagents and solvents were purified according to known procedures. ${ }^{1}$ Thin layer chromatographic (TLC) analyses were performed on silica gel plates Merck $60 \mathrm{~F}_{254}$. Column chromatographic purifications ("flash chromatography", FC) were performed as described. ${ }^{2}$ Melting points were determined using a Büchi SMP-20 apparatus and are uncorrected. Optical rotations were measured with a JASCO DIP-1000 polarimeter. ${ }^{1} \mathrm{H}$ NMR and ${ }^{13} \mathrm{C}$ NMR spectra were obtained using a Bruker ARX 300 instrument at 300.1 and $75.4 \mathrm{MHz}$, respectively. Unless otherwise stated, $\mathrm{CDCl}_{3}$ was used as solvent. Infrared (IR) spectra were recorded with a Perkin-Elmer FT-IR 16 PC instrument either from $\mathrm{KBr}$ plates or neat. Ultraviolet (UV) spectra were obtained from alcoholic solutions utilizing a Shimadzu UV160A or an ATI UNICAM UV 3 instrument. Mass spectra were achieved by electron impact (EI) method using a Finnigan MAT SSQ 710 instrument. Elemental analyses were performed on a LECO CHNS-932 instrument.

General procedure 1 (GP 1): Preparation of $N$-sulfamoylcarbamates. To a solution of $0.05 \mathrm{~mol}$ of the benzyl alcohol in THF $(100 \mathrm{~mL})$ was added $0.05 \mathrm{~mol}$ of CSI in such a way that the internal temperature was maintained below $-5^{\circ} \mathrm{C}\left(-60{ }^{\circ} \mathrm{C}\right.$ for alkyl substituents $)$. The mixture was stirred for $30 \mathrm{~min}$ at $-5{ }^{\circ} \mathrm{C}\left(-60^{\circ} \mathrm{C}\right)$ and $0.1 \mathrm{~mol}$ of the aniline was added at that 
temperature. The solution was stirred for $2 \mathrm{~h}$ by which time the temperature raised to $5-10{ }^{\circ} \mathrm{C}$ (raised to $-30{ }^{\circ} \mathrm{C}$ ). An aqueous solution of $1 \mathrm{~N} \mathrm{HCl}$ was added until $\mathrm{pH} \sim 2$ was obtained. The aqueous layer was extracted with $\mathrm{Et}_{2} \mathrm{O}(3 \times 20 \mathrm{~mL})$. The combined organic extracts were dried over $\mathrm{MgSO}_{4}$, filtered, and concentrated under reduced pressure. The residue was redissolved, filtered through a plug of silica gel and recrystallized from an appropriate solvent.

Benzyl $N$-phenyl-sulfamoylcarbamate (1). According to GP 1, benzyl alcohol $(5.41 \mathrm{~g}$, $50 \mathrm{mmol})$ was reacted with CSI $(4.4 \mathrm{~mL}, 7.2 \mathrm{~g}, 51 \mathrm{mmol})$ and aniline $(10.0 \mathrm{~mL}, 10.2 \mathrm{~g}, 110$ $\mathrm{mmol})$ in $\mathrm{THF}(100 \mathrm{~mL})$ at $-10{ }^{\circ} \mathrm{C}$.

Recrystallized from EtOAc and light petroleum.

Yield of 1: $11.9 \mathrm{~g}$ (78\%). Colorless needles. M.p. 140-141 ${ }^{\circ} \mathrm{C} .-\mathrm{IR}$ (KBr): $\widetilde{v}$ 3237, 1716, 1600, 1452, 1158, $764 \mathrm{~cm}^{-1} .-{ }^{1} \mathrm{H}$ NMR: $7.32-7.11\left(\mathrm{~m}, 10 \mathrm{H}, \mathrm{H}_{\mathrm{Ar}}\right) ; 5.11\left(\mathrm{~s}, 2 \mathrm{H}, \mathrm{CH}_{2} \mathrm{O}\right) ; 4.12$ (br. s, 2H, NH). $-{ }^{13} \mathrm{C}$ NMR: 151.69 (s), 136.23 (s), 134.79 (s), 128.90 (d), 128.19 (d), 128.10 (d), 127.69 (d), 124.93 (d), 120.91 (d), 67.49 (t). - MS (70eV): m/z (\%) 306 (27), 262 (8), 198 (2), 181 (57), 91 (100). - UV (EtOH): $\quad \max$ ( ) 231 (7700), 202 (28500) nm. elemental analysis (\%): calcd. for $\mathrm{C}_{14} \mathrm{H}_{14} \mathrm{~N}_{2} \mathrm{O}_{4} \mathrm{~S}$ (306.34): C 54.89, H 4.61, N 9.14, S 10.47; found C 54.75, H 4.64, N 9.11, S 10.51 .

2,4,6-Trimethylbenzyl $N$-phenyl-sulfamoylcarbamate (2). According to GP 1, 2,4,6trimethylbenzyl alcohol $(3.75 \mathrm{~g}, 25 \mathrm{mmol})$ was reacted with CSI $(2.3 \mathrm{~mL}, 3.74 \mathrm{~g}, 26 \mathrm{mmol})$ and aniline $(5.6 \mathrm{~mL}, 5.7 \mathrm{~g}, 61 \mathrm{mmol})$ in $\operatorname{THF}(80 \mathrm{~mL})$ at $-9{ }^{\circ} \mathrm{C}$.

Recrystallized from EtOAc and light petroleum.

Yield of 2: $7.8 \mathrm{~g}$ (90\%). Colorless solid. M.p. $172{ }^{\circ} \mathrm{C}$. - IR (KBr): $\widetilde{v}$ 3272, 2923, 1725, 1600, 1439, 1155, $843 \mathrm{~cm}^{-1} .{ }^{1}{ }^{\mathrm{H}} \mathrm{NMR}\left(\mathrm{MeOH}_{-} \mathrm{d}_{4}\right): \quad 7.28-7.23\left(\mathrm{~m}, 2 \mathrm{H}, \mathrm{H}_{\mathrm{Ar}}\right) ; 7.16-7.08$ (m, 3H, $\left.\mathrm{H}_{\mathrm{Ar}}\right) ; 6.80\left(\mathrm{~s}, 2 \mathrm{H}, \mathrm{H}_{\mathrm{Ar}}\right) ; 5.15$ (s, 2H, $\left.\mathrm{CH}_{2} \mathrm{O}\right) ; 2.22$ (s, 9H, $\left.\mathrm{CH}_{3}\right) .-{ }^{13} \mathrm{C} \mathrm{NMR}\left(\mathrm{MeOH}-\mathrm{d}_{4}\right)$ : 153.33 (s), 139.63 (s), 139.36 (s), 138.61 (s), 130.13 (d), 129.89 (d), 129.73 (s), 125.79 (d), 121.93 (d), 63.58 (t), 21.08 (q), 19.54 (q). - MS (70eV): m/z (\%) 348 (0.7), 304 (0.5), 224 (1), 133 (100). - UV (EtOH): $\quad \max (~)=225$ (15200), 203 (66700) nm. - elemental analysis (\%): calcd. for $\mathrm{C}_{17} \mathrm{H}_{20} \mathrm{~N}_{2} \mathrm{O}_{4} \mathrm{~S}$ (348.42): C 58.60, H 5.79, N 8.04, S 9.20; found C 58.29, H 6.01, N 7.97, S 9.22. 


\section{2-Methoxycarbonylmethoxybenzyl $N$-(4-chlorophenyl)-sulfamoylcarbamate (3).}

According to GP 1, 2-Methoxycarbonylmethoxybenzyl alcohol (8.05 g, $41 \mathrm{mmol})$ was reacted with CSI $(4.0 \mathrm{~mL}, 5.80 \mathrm{~g}, 41 \mathrm{mmol})$ and $p$-chloroaniline $(10.5 \mathrm{~g}, 82 \mathrm{mmol})$ in THF $(80 \mathrm{~mL})$ at $-5{ }^{\circ} \mathrm{C}$.

Recrystallized from EtOAc and light petroleum.

Yield of 3: $12.6 \mathrm{~g}$ (72\%). Colorless solid. M.p. $153{ }^{\circ} \mathrm{C} .-{ }^{1} \mathrm{H}$ NMR: 8.01 (br. s, 2H, NH); $7.32\left(\mathrm{td}, J=7.9,1.6,1 \mathrm{H}, \mathrm{H}_{\mathrm{Ar}}\right) ; 7.25\left(\mathrm{dd}, J=7.4,1.6,1 \mathrm{H}, \mathrm{H}_{\mathrm{Ar}}\right) ; 7.14\left(\mathrm{~d}, J=8.7 \mathrm{~Hz}, 2 \mathrm{H}, \mathrm{H}_{\mathrm{Ar}}\right)$; $6.96\left(\mathrm{~m}, 1 \mathrm{H}, \mathrm{H}_{\mathrm{Ar}}\right) ; 6.95\left(\mathrm{~d}, J=8.6 \mathrm{~Hz}, 2 \mathrm{H}, \mathrm{H}_{\mathrm{Ar}}\right) ; 6.74\left(\mathrm{~d}, J=8.3 \mathrm{~Hz}, 1 \mathrm{H}, \mathrm{H}_{\mathrm{Ar}}\right) ; 5.23(\mathrm{~s}, 2 \mathrm{H}$, $\mathrm{CH}_{2} \mathrm{O}$ ); 4.65 (s, 2H, $\mathrm{CH}_{2} \mathrm{O}$ ); 3.74 (s, 3H, $\left.\mathrm{CH}_{3} \mathrm{O}\right) .-{ }^{13} \mathrm{C}$ NMR: 169.67 (s), 156.10 (s), 151.18(s), 134.40 (s), 131.28 (s), 131.21 (d), 130.53 (d), 129.33 (d), 123.37 (s), 123.19 (d), 121.66 (d), 111.33 (d) 64.91 (t), 64.37 (t), 52.44 (q). - MS (70eV): m/z (\%) 430 (1), 428 (3), 234 (9), 232 (27), 208 (2), 206 (7), 179 (100), 151 (13), 126 (61), 124 (52).

2,4-Dichlorobenzyl $N$-(4-chlorophenyl)-sulfamoylcarbamate (4). According to GP 1, 2,4dichlorobenzyl alcohol $(8.47 \mathrm{~g}, 48 \mathrm{mmol})$ was reacted with CSI $(4.3 \mathrm{~mL}, 7.0 \mathrm{~g}, 49 \mathrm{mmol})$ and $p$-chloroaniline $(12.5 \mathrm{~g}, 98 \mathrm{mmol})$ in $\mathrm{THF}(100 \mathrm{~mL})$ at $-20{ }^{\circ} \mathrm{C}$.

Recrystallized from EtOAc and light petroleum.

Yield of 4: $17.1 \mathrm{~g}$ (87\%). Colorless solid. M.p. $160{ }^{\circ} \mathrm{C} .-\mathrm{IR}(\mathrm{KBr}): \widetilde{v} 3244,1714,1593$, 1451, 1166, 861, $598 \mathrm{~cm}^{-1} .{ }^{1} \mathrm{H}$ NMR (DMSO-d 6 ): 11.81 (br. s, 1H, NH); 10.76 (s, 1H, $\mathrm{NH}) ; 7.63\left(\mathrm{~s}, 1 \mathrm{H}, \mathrm{H}_{\mathrm{Ar}}\right) ; 7.37\left(\mathrm{~m}, 4 \mathrm{H}, \mathrm{H}_{\mathrm{Ar}}\right) ; 7.16\left(\mathrm{~d}, J=8 \mathrm{~Hz}, 2 \mathrm{H}, \mathrm{H}_{\mathrm{Ar}}\right) ; 5.15\left(\mathrm{~s}, 2 \mathrm{H}, \mathrm{CH}_{2} \mathrm{O}\right)$. ${ }^{13}$ C NMR (DMSO-d D $_{6}$ ) 150.80 (s), 136.44 (s), 133.99 (s), 133.70 (s), 132.18 (s), 131.42 (d), 129.08 (d), 128.97 (d), 127.92 (s), 127.49 (d), 120.80 (d), 63.70 (t). - MS (70eV): m/z (\%) 412 (0.7), 410 (1.5), 408 (1.4), 318 (1.1), 316 (1.5), 287 (0.8), 285 (2), 283 (1.7), 234 (5), 232 (16), 160 (58), 159 (100), 126 (54), 99 (39). - UV (EtOH): $\quad \max ($ ) = 241 (11800), 203 (53500) nm. - elemental analysis (\%): calcd. for $\mathrm{C}_{14} \mathrm{H}_{11} \mathrm{Cl}_{3} \mathrm{~N}_{2} \mathrm{O}_{4} \mathrm{~S}$ (409.68): C 41.05, H 2.71, N 6.84, S 7.83; found C 41.08, H 2.81, N 6.85, S 7.80.

General procedure 2 (GP 2): The Friedel-Crafts alkylation. A suspension of $2 \mathrm{mmol}$ of $\mathrm{N}$-Sulfamoylcarbamate in the aromatic solvent (2-4 mL) was heated to $110-125^{\circ} \mathrm{C}$. Above $105{ }^{\circ} \mathrm{C}$ a gas evolution started and the solid dissolved slowly. A colored precipitation was formed during heating and after $5 \mathrm{~h}$ at $110-125^{\circ} \mathrm{C}$, no starting material was detected (TLC 
analysis). The solvent was removed under reduced pressure, when possible, and the residue purified by FC on silica gel and recrystallization.

Methyl 2-[2-(2,4,6-trimethylbenzyl)-phenoxy]-acetate (6). According to GP 2, sulfamoylcarbamate $3(2.00 \mathrm{~g}, 4.67 \mathrm{mmol})$ was heated in mesitylene $(4.0 \mathrm{~mL}, 3,46 \mathrm{~g}$, $29 \mathrm{mmol})$ at $130{ }^{\circ} \mathrm{C}$.

$\mathrm{FC}\left(\mathrm{SiO}_{2}, 3\right.$ x $\left.30 \mathrm{~cm}, 11 \% \mathrm{EtOAc/light} \mathrm{petroleum}\right)$.

Yield of 6: $0.71 \mathrm{~g}(51 \%)$. Colorless solid. M.p. $78-79{ }^{\circ} \mathrm{C}$. - IR (KBr): $\widetilde{v}$ 2926, 1763, 1600, 1584, 1488, 1454, 1203, 1115, $749 \mathrm{~cm}^{-1} .-{ }^{1} \mathrm{H}$ NMR (DMSO-d 6 ): 7.12 (t, $J=7.7 \mathrm{~Hz}, 1 \mathrm{H}$, $\left.\mathrm{H}_{\mathrm{Ar}}\right) ; 6.91\left(\mathrm{~d}, J=8.5 \mathrm{~Hz}, 1 \mathrm{H}, \mathrm{H}_{\mathrm{Ar}}\right) ; 6.88\left(\mathrm{~s}, 2 \mathrm{H}, \mathrm{H}_{\mathrm{Ar}}\right) ; 6.76\left(\mathrm{t}, J=7.5 \mathrm{~Hz}, 1 \mathrm{H}, \mathrm{H}_{\mathrm{Ar}}\right) ; 6.38(\mathrm{~d}$, $\left.J=7.5 \mathrm{~Hz}, 1 \mathrm{H}, \mathrm{H}_{\mathrm{Ar}}\right) ; 4.91\left(\mathrm{~s}, 2 \mathrm{H}, \mathrm{CH}_{2} \mathrm{O}\right) ; 3.91\left(\mathrm{~s}, 2 \mathrm{H}, \mathrm{CH}_{2}\right) ; 3.72\left(\mathrm{~s}, 3 \mathrm{H}, \mathrm{CH}_{3} \mathrm{O}\right) ; 2.24(\mathrm{~s}, 3 \mathrm{H}$, $\left.\mathrm{CH}_{3}\right) ; 2.09$ (s, 6H, $\left.\mathrm{CH}_{3}\right) .-{ }^{13} \mathrm{C}$ NMR (DMSO-d $)$ ): 169.41 (s), 155.49 (s), 136.55 (s), 134.90 (s), 132.99 (s), 128.66 (d), 127.88 (s), 127.00 (d), 126.86 (d), 121.05 (d), 111.35 (d), 64.85 (t), 51.84 (q), 27.87 (t), 20.56 (q), 19.47 (q). - MS (70eV): m/z (\%) 298 (100), 283 (13), 225 (18), 209 (37), 179 (16), 133 (21). - UV (EtOH): $\quad \max ($ ) = 271 (3800), 203 (62000) nm. elemental analysis (\%): calcd. for $\mathrm{C}_{19} \mathrm{H}_{22} \mathrm{O}_{3}$ (298.39): C 76.48, $\mathrm{H}$ 7.43; found C 76.23, H 7.37.

2,4,6-Trimethylbenzylbenzene (7). According to GP 2, sulfamoylcarbamate 1 (0.92 g, 3.00 mmol) was heated in mesitylene $(3.0 \mathrm{~mL}, 2.59 \mathrm{~g}, 22 \mathrm{mmol})$ at $127^{\circ} \mathrm{C}$.

$\mathrm{FC}\left(\mathrm{SiO}_{2}, 3\right.$ x $20 \mathrm{~cm}, 0-100 \%$ EtOAc/hexane).

Yield of 1: $0.45 \mathrm{~g}$ (71\%). Colorless oil. Yield of dibenzylmesitylene: $0.05 \mathrm{~g}$ (9\%). Pale yellow oil.

7: ${ }^{1} \mathrm{H}$ NMR: $7.23-7.18\left(\mathrm{~m}, 2 \mathrm{H}, \mathrm{H}_{\mathrm{Ar}}\right) ; 7.12\left(\mathrm{~m}, 1 \mathrm{H}, \mathrm{H}_{\mathrm{Ar}}\right) ; 7.00\left(\mathrm{~d}, J=7.4 \mathrm{~Hz}, 2 \mathrm{H}, \mathrm{H}_{\mathrm{Ar}}\right) ; 6.88$ $\left(\mathrm{s}, 2 \mathrm{H}, \mathrm{H}_{\mathrm{Ar}}\right) ; 4.00\left(\mathrm{~s}, 2 \mathrm{H}, \mathrm{CH}_{2}\right) ; 2.28\left(\mathrm{~s}, 3 \mathrm{H}, \mathrm{CH}_{3}\right) ; 2.19\left(\mathrm{~s}, 6 \mathrm{H}, \mathrm{CH}_{3}\right) .-{ }^{13} \mathrm{C} \mathrm{NMR}$ : 140.06 (s), 136.97 (s), 135.61 (s), 133.75 (s), 128.86 (d), 128.31 (d), 127.81 (d), 125.62 (d), 34.67 (t), 20.88 (q), 20.10 (q). - MS (70eV): m/z (\%) 210 (100), 195 (69), 165 (14).

dibenzylmesitylene: ${ }^{1} \mathrm{H}$ NMR: $7.25-7.20\left(\mathrm{~m}, 4 \mathrm{H}, \mathrm{H}_{\mathrm{Ar}}\right) ; 7.14\left(\mathrm{~m}, 2 \mathrm{H}, \mathrm{H}_{\mathrm{Ar}}\right) ; 7.03-7.00(\mathrm{~m}$, $\left.4 \mathrm{H}, \mathrm{H}_{\mathrm{Ar}}\right) ; 6.96\left(\mathrm{~s}, 1 \mathrm{H}, \mathrm{H}_{\mathrm{Ar}}\right) ; 4.07\left(\mathrm{~s}, 4 \mathrm{H}, \mathrm{CH}_{2}\right) ; 2.24\left(\mathrm{~s}, 6 \mathrm{H}, \mathrm{CH}_{3}\right) ; 2.08\left(\mathrm{~s}, 3 \mathrm{H}, \mathrm{CH}_{3}\right)$. ${ }^{13}$ C NMR: 140.23 (s), 136.34 (s), 135.13 (s), 134.75 (s), 129.96 (d), 128.33 (d), 127.82 (d), 125.62 (d), 35.46 (t), 20.27 (q), 16.21 (q). - MS (70eV): m/z (\%) 300 (100), 285 (10), 209 (65), 179 (23), 165 (11), 91 (35). 
Dimesitylmethane (8). According to GP 2, sulfamoylcarbamate 2 (0.69 g, $1.98 \mathrm{mmol})$ was heated in mesitylene $(1.7 \mathrm{~mL}, 1.47 \mathrm{~g}, 12 \mathrm{mmol})$ at $125^{\circ} \mathrm{C}$.

$\mathrm{FC}\left(\mathrm{SiO}_{2}, 3\right.$ x $20 \mathrm{~cm}, 0-20 \%$ EtOAc/light petroleum).

Yield of 8: $0.10 \mathrm{~g}(20 \%)$. Colorless oil. ${ }^{1} \mathrm{H}$ NMR: $6.88\left(\mathrm{~s}, 4 \mathrm{H}, \mathrm{H}_{\mathrm{Ar}}\right) ; 4.10\left(\mathrm{~s}, 2 \mathrm{H}, \mathrm{CH}_{2}\right) ; 2.35$ (s, 6H, $\left.\mathrm{CH}_{3}\right) ; 2.19$ (s, 12H, $\left.\mathrm{CH}_{3}\right) .-{ }^{13} \mathrm{C} \mathrm{NMR}: 136.65$ (s), 134.87 (s), 134.78 (s), 129.29 (d), 30.98 (t), 20.76 (q), 20.69 (q). - MS (70eV): m/z (\%) 252 (100), 237 (33), 132 (82).

1,3-Dichlor-4-(2,4,6-trimethylbenzyl)-benzene (9). According to GP 8, sulfamoylcarbamate $4(1.00 \mathrm{~g}, 2.44 \mathrm{mmol})$ was heated in mesitylene $(3 \mathrm{~mL}, 1.29 \mathrm{~g}, 22 \mathrm{mmol})$ at $130^{\circ} \mathrm{C}$. $\mathrm{FC}\left(\mathrm{SiO}_{2}, 3 \times 30 \mathrm{~cm}, 6 \% \mathrm{EtOAc} /\right.$ light petroleum $)$.

Yield of 9: $0.60 \mathrm{~g}(88 \%)$. Brownish crystals. M.p. $93.7{ }^{\circ} \mathrm{C} .-{ }^{1} \mathrm{H}$ NMR (DMSO-d $\left.)_{6}\right): 7.39$ (d, $\left.J=1.5 \mathrm{~Hz}, 1 \mathrm{H}, \mathrm{H}_{\mathrm{Ar}}\right) ; 6.99\left(\mathrm{dd}, J=8.3,1.5 \mathrm{~Hz}, 1 \mathrm{H}, \mathrm{H}_{\mathrm{Ar}}\right) ; 6.69\left(\mathrm{~s}, 2 \mathrm{H}, \mathrm{H}_{\mathrm{Ar}}\right) ; 6.48(\mathrm{~d}, J=$ $\left.8.3 \mathrm{~Hz}, 1 \mathrm{H}, \mathrm{H}_{\mathrm{Ar}}\right) ; 3.96\left(\mathrm{~s}, 2 \mathrm{H}, \mathrm{CH}_{2}\right) ; 2.29\left(\mathrm{~s}, 3 \mathrm{H}, \mathrm{CH}_{3}\right) ; 2.12\left(\mathrm{~s}, 6 \mathrm{H}, \mathrm{CH}_{3}\right) .-{ }^{13} \mathrm{C} \mathrm{NMR}$ (DMSO-d ${ }_{6}$ ): 137.10 (s), 136.20 (s), 136.13 (s), 134.84 (s), 132.06 (s), 131.99 (s), 129.01 (d), 128.83 (d), 127.01 (d), 31.84 (t), 20.90 (q), 19.79 (q). - MS (70eV): m/z (\%) 280 (63), 278 (100), 265 (32), 263 (74), 192 (22), 178 (15), 132 (32), 91 (15).

Methyl 2-[2-(2-methylbenzyl)-phenoxy]-acetate (o-10) and methyl 2-[2-(4-methylbenzyl)phenoxy]-acetate ( $p$-10). According to GP 2, sulfamoylcarbamate 3 (2.00 g, $4.67 \mathrm{mmol})$ was heated in toluene $(5.0 \mathrm{~mL}, 4.32 \mathrm{~g}, 47 \mathrm{mmol})$ at $111^{\circ} \mathrm{C}$.

$\mathrm{FC}\left(\mathrm{SiO}_{2}, 3\right.$ x $30 \mathrm{~cm}, 11 \%$ EtOAc/light petroleum).

Yield of $p$-10 and $o-10: 0.48 \mathrm{~g}(38 \%)$. Colorless oil. Ratio of $p-\mathbf{1 0}$ and $o-\mathbf{1 0}=1: 2.5$.

$o$-10: ${ }^{1} \mathrm{H}$ NMR: $7.20-6.69\left(\mathrm{~m}, 8 \mathrm{H}, \mathrm{H}_{\mathrm{Ar}}\right) ; 4.59\left(\mathrm{~s}, 2 \mathrm{H}, \mathrm{CH}_{2} \mathrm{O}\right) ; 4.00\left(\mathrm{~s}, 2 \mathrm{H}, \mathrm{CH}_{2}\right) ; 3.77(\mathrm{~s}, 3 \mathrm{H}$, $\mathrm{CH}_{3} \mathrm{O}$ ); 2.29 (s, 3H, $\left.\mathrm{CH}_{3}\right) .-{ }^{13} \mathrm{C}$ NMR: 169.45 (s), 155.46 (s), 137.60 (s), 135.12 (s), 130.53 (d), 129.79 (s), 128.85 (d), 127.17 (d), 121.55 (d), 111.33 (d), 65.41 (t), 52.02 (q), 35.32 (t), 20.92 (q). - MS (70eV): m/z (\%) 270 (100), 255 (4), 210 (13), 197 (85), 181 (76), 165 (29), 152 (12), 105 (21).

p-10: ${ }^{1} \mathrm{H}$ NMR: 4.63 (s, 2H, $\left.\mathrm{CH}_{2} \mathrm{O}\right) ; 4.03\left(\mathrm{~s}, 2 \mathrm{H}, \mathrm{CH}_{2}\right) ; 2.25$ (s, 3H, $\left.\mathrm{CH}_{3}\right) .-{ }^{13} \mathrm{C}$ NMR: 155.56 (s), 138.41 (s), 137.00 (s), 130.56 (d), 130.00 (d), 129.97 (d), 129.27 (s), 127.04 (d), 126.13 (d), 125.78 (d), 121.53 (d), 110.06 (d), 65.38 (t), 32.95 (t), 19.44 (q). 
Methyl 2-[2-(2,4-dimethylbenzyl)-phenoxy]-acetate (o,p-11) and methyl 2-[2-(2,6dimethylbenzyl)-phenoxy]-acetate (o,p-11). According to GP 2, sulfamoylcarbamate 3 $(2.00 \mathrm{~g}, 4.67 \mathrm{mmol})$ was heated in $m$-xylene $(5.0 \mathrm{~mL}, 4.34 \mathrm{~g}, 41 \mathrm{mmol})$ at $138{ }^{\circ} \mathrm{C}$.

$\mathrm{FC}\left(\mathrm{SiO}_{2}, 3\right.$ x $\left.30 \mathrm{~cm}, 11 \% \mathrm{EtOAc/light} \mathrm{petroleum}\right)$.

Yield of $o, p-11$ and $o, o-11: 0.82 \mathrm{~g}(62 \%)$. Colorless oil.

Ratio of $o, p-\mathbf{1 1}$ and $o, o-11=6: 1$.

$o, p-11:{ }^{1} \mathrm{H}$ NMR: $\quad 7.14-6.50\left(\mathrm{~m}, 7 \mathrm{H}, \mathrm{H}_{\mathrm{Ar}}\right) ; 4.61\left(\mathrm{~s}, 2 \mathrm{H}, \mathrm{CH}_{2} \mathrm{O}\right) ; 3.99\left(\mathrm{~s}, 2 \mathrm{H}, \mathrm{CH}_{2}\right) ; 3.74(\mathrm{~s}$, $\left.3 \mathrm{H}, \mathrm{CH}_{3} \mathrm{O}\right) ; 2.28\left(\mathrm{~s}, 3 \mathrm{H}, \mathrm{CH}_{3}\right) ; 2.21\left(\mathrm{~s}, 3 \mathrm{H}, \mathrm{CH}_{3}\right) .-{ }^{13} \mathrm{C} \mathrm{NMR}: 169.38$ (s), 155.55 (s), 136.47 (s), 135.46 (s), 135.28 (s), 130.81 (d), 129.92 (d), 129.77 (d), 129.65 (s), 126.91 (d), 126.40 (d), 121.48 (d), 111.02 (d), 65.37 (t), 51.95 (q), 32.51 (t), 20.82 (q), 19.33 (q). - MS (70eV): m/z (\%) 284 (100), 269 (7), 211 (54), 195 (73), 179 (52), 165 (20), 119 (16).

o,o-11: ${ }^{1} \mathrm{H}$ NMR: $4.70\left(\mathrm{~s}, 2 \mathrm{H}, \mathrm{CH}_{2} \mathrm{O}\right) ; 4.06\left(\mathrm{~s}, 2 \mathrm{H}, \mathrm{CH}_{2}\right) ; 3.77\left(\mathrm{~s}, 3 \mathrm{H}, \mathrm{CH}_{3} \mathrm{O}\right) ; 2.19(\mathrm{~s}, 6 \mathrm{H}$, $\mathrm{CH}_{3}$ ). $-{ }^{13} \mathrm{C}$ NMR: 155.61 (s), 137.33 (s), 136.37 (s), 128.54 (s), 127.88 (d), 127.76 (d), 126.63 (d), 126.09 (s), 121.55 (d), 110.56 (d), 65.30 (t), 52.01 (q), 28.61 (t), 19.85 (q).

\section{Methyl 2-(2- -napthylmethyl)-phenoxy-acetate ( -12) and methyl 2-(2- -} napthylmethyl)-phenoxy-acetate (-12). According to GP 2, sulfamoylcarbamate 3 $(2.00 \mathrm{~g}, 4.67 \mathrm{mmol})$ was heated in naphthalene $(5.0 \mathrm{~g}, 39 \mathrm{mmol})$ at $130{ }^{\circ} \mathrm{C}$.

$\mathrm{FC}\left(\mathrm{SiO}_{2}, 3\right.$ x $\left.30 \mathrm{~cm}, 14 \% \mathrm{EtOAc/light} \mathrm{petroleum}\right)$.

Yield of $\mathbf{- 1 2}$ and $\mathbf{- 1 2}$ : $0.81 \mathrm{~g}(57 \%)$. Colorless oil. Ratio of $\mathbf{- 1 2}$ and $\mathbf{- 1 2}=4: 1$.

-12: ${ }^{1} \mathrm{H}$ NMR: $8.01\left(\mathrm{~m}, 1 \mathrm{H}, \mathrm{H}_{\mathrm{Ar}}\right) ; 7.78\left(\mathrm{~m}, 1 \mathrm{H}, \mathrm{H}_{\mathrm{Ar}}\right) ; 7.68\left(\mathrm{~d}, J=7.7 \mathrm{~Hz}, 1 \mathrm{H}, \mathrm{H}_{\mathrm{Ar}}\right) ; 7.40$ $7.24\left(\mathrm{~m}, 4 \mathrm{H}, \mathrm{H}_{\mathrm{Ar}}\right) ; 7.06\left(\mathrm{~m}, 1 \mathrm{H}, \mathrm{H}_{\mathrm{Ar}}\right) ; 6.88-6.63\left(\mathrm{~m}, 3 \mathrm{H}, \mathrm{H}_{\mathrm{Ar}}\right) ; 4.59\left(\mathrm{~s}, 2 \mathrm{H}, \mathrm{CH}_{2} \mathrm{O}\right) ; 4.48(\mathrm{~s}$, 2H, $\mathrm{CH}_{2}$ ); 3.66 (s, 3H, CH $\mathrm{CH}_{2} \mathrm{O}$ ) $-{ }^{13} \mathrm{C}$ NMR: 169.29 (s), 155.24 (s), 136.26 (s), 133.68 (s), 132.16 (s), 130.22 (d), 129.47 (s), 128.39 (d), 127.10 (d), 127.05 (d), 126.78 (d), 125.70 (d), 125.40 (d), 125.31 (d), 124.31 (d), 121.46 (d), 110.97 (d), 65.25 (t), 51.88 (q), 32.36 (t).

-12: ${ }^{1} \mathrm{H}$ NMR: $4.50\left(\mathrm{~s}, 2 \mathrm{H}, \mathrm{CH}_{2} \mathrm{O}\right) ; 4.18\left(\mathrm{~s}, 2 \mathrm{H}, \mathrm{CH}_{2}\right) ; 3.64\left(\mathrm{~s}, 3 \mathrm{H}, \mathrm{CH}_{2} \mathrm{O}\right) .-{ }^{13} \mathrm{C}$ NMR: 169.18 (s), 155.47 (s), 138.19 (s), 133.46 (s), 131.87 (s), 130.62 (d), 130.05 (s), 127.73 (d), 127.60 (d), 127.39 (d), 127.30 (d), 125.60 (d), 124.97 (d), 121.51 (d), 111.28 (d), 51.84 (q), $35.91(\mathrm{t})$. 
1-Benzyl-naphthalene ( -13) and 2-benzyl-naphthalene ( -13). Similarly to GP 2, sulfamoylcarbamate 1 (307 mg, $1.00 \mathrm{mmol}$ ) was heated with naphthalene (195 mg, $1.52 \mathrm{mmol})$ in octane $(3 \mathrm{~mL})$ at $127^{\circ} \mathrm{C}$.

$\mathrm{FC}\left(\mathrm{SiO}_{2}, 3 \times 20 \mathrm{~cm}, 14 \% \mathrm{EtOAc} /\right.$ hexane$)$.

Yield of $\mathbf{- 1 3}$ and $\quad \mathbf{- 1 3}: 23 \mathrm{mg}(10 \%)$. Colorless oil. Ratio of $\quad \mathbf{- 1 3}$ and $\quad \mathbf{- 1 3}=8: 1$.

-13: ${ }^{1} \mathrm{H}$ NMR: $7.99\left(\mathrm{~m}, 1 \mathrm{H}, \mathrm{H}_{\mathrm{Ar}}\right) ; 7.85\left(\mathrm{~m}, 1 \mathrm{H}, \mathrm{H}_{\mathrm{Ar}}\right) ; 7.75\left(\mathrm{~m}, 1 \mathrm{H}, \mathrm{H}_{\mathrm{Ar}}\right) ; 7.45-7.38(\mathrm{~m}, 3 \mathrm{H}$, $\left.\mathrm{H}_{\mathrm{Ar}}\right) ; 7.29-7.17\left(\mathrm{~m}, 6 \mathrm{H}, \mathrm{H}_{\mathrm{Ar}}\right) ; 4.44\left(\mathrm{~s}, 2 \mathrm{H}, \mathrm{CH}_{2}\right)$. - MS (70eV): m/z (\%) $218(100), 202(32)$, 189 (9), 141 (24), 115 (15).

-13: ${ }^{1} \mathrm{H}$ NMR: $4.13\left(\mathrm{~s}, 2 \mathrm{H}, \mathrm{CH}_{2}\right)$.

Diphenylmethane 15. Similarly to GP 2, sulfamoylcarbamate 1 (0.61 g, $2.0 \mathrm{mmol})$ was heated in benzene $(5.0 \mathrm{~mL}, 4.37 \mathrm{~g}, 56 \mathrm{mmol})$ at $120{ }^{\circ} \mathrm{C}$ in a closed vessel.

$\mathrm{FC}\left(\mathrm{SiO}_{2}, 2\right.$ x $22 \mathrm{~cm}, 0-20 \%$ EtOAc/hexane).

Yield of 15: $87 \mathrm{mg}$ (25\%). Colorless oil. ${ }^{1} \mathrm{H}$ NMR: $\quad$ 7.42-7.30 (m, 10H, $\left.\mathrm{H}_{\mathrm{Ar}}\right) ; 4.10(\mathrm{~s}, 2 \mathrm{H}$, $\left.\mathrm{CH}_{2}\right) .-{ }^{13} \mathrm{C}$ NMR: 141.08 (s), 128.83 (d), 128.42 (d), 126.03 (d), 41.91 (t). - MS (70eV): $m / z(\%) 167$ (100), 149 (70).

3-Benzyl-1H-indole 16. According to GP 2, sulfamoylcarbamate 1 (0.31 g, $1.0 \mathrm{mmol})$ was heated in indole $(0.35 \mathrm{~g}, 3.0 \mathrm{mmol})$ at $125^{\circ} \mathrm{C}$.

$\mathrm{FC}\left(\mathrm{SiO}_{2}, 2\right.$ x $25 \mathrm{~cm}, 25-100 \%$ EtOAc/hexane $)$.

Yield of 16: $50 \mathrm{mg}$ (25\%). Colorless oil. ${ }^{1} \mathrm{H}$ NMR: 7.49 (d, J=7.9 Hz, 1H, $\mathrm{H}_{\mathrm{Ar}}$ ); 6.70 (br. s, $\left.1 \mathrm{H}, \mathrm{H}_{\mathrm{Ar}}\right) ; 4.05$ (s, 2H, CH2). - MS (70eV): m/z (\%) 207 (100), 206 (37), 130 (34).

2-Benzyl-4-bromoanisole (17). According to GP 2, sulfamoylcarbamate 1 (0.68 g, $2.2 \mathrm{mmol})$ was heated in 4-bromoanisole $(2.0 \mathrm{~mL}, 3.0 \mathrm{~g}, 16 \mathrm{mmol})$ at $128{ }^{\circ} \mathrm{C}$.

$\mathrm{FC}\left(\mathrm{SiO}_{2}, 3 \times 20 \mathrm{~cm}, 0-1 \% \mathrm{Et}_{2} \mathrm{OAc} /\right.$ light petroleum $)$.

Yield of 17: $0.25 \mathrm{~g}(41 \%)$. Colorless oil. ${ }^{1} \mathrm{H}$ NMR: $7.23-7.14\left(\mathrm{~m}, 7 \mathrm{H}, \mathrm{H}_{\mathrm{Ar}}\right) ; 6.65(\mathrm{~d}, J=$ $8.6 \mathrm{~Hz}, 1 \mathrm{H}, \mathrm{H}_{\mathrm{Ar}}$ ); 3.89 (s, 2H, $\mathrm{CH}_{2}$ ); 3.71 (s, 3H, $\left.\mathrm{CH}_{3} \mathrm{O}\right) .-{ }^{13} \mathrm{C} \mathrm{NMR:} 156.35$ (s), 139.98 (s), 132.78 (d), 131.93 (s), 129.94 (d), 128.83 (d), 128.32 (d), 126.00 (d), 112.63 (s), 111.98 (d), 55.44 (q), 35.56 (t). - MS (70eV): m/z (\%) 278 (99), 276 (100), 263 (10), 261 (11), 197 (50), 182 (24), 181 (28), 165 (41), 152 (27). 
2-Benzyl-4-chlorophenol (18) and 4-Chlorophenylbenzylether (21). According to GP 2, sulfamoylcarbamate $1(0.61 \mathrm{~g}, 2.0 \mathrm{mmol})$ was heated in 4-chlorophenol $(2.0 \mathrm{~mL}, 2.61 \mathrm{~g}$, $20 \mathrm{mmol})$ at $120^{\circ} \mathrm{C}$.

$\mathrm{FC}\left(\mathrm{SiO}_{2}, 2\right.$ x $22 \mathrm{~cm}, 20-50 \%$ EtOAc/hexane).

Yield of 18: $83 \mathrm{mg}$ (19\%). Yield of 21: $110 \mathrm{mg}$ (25\%). Colorless oils.

18: ${ }^{1} \mathrm{H}$ NMR: $\quad 7.32-7.11\left(\mathrm{~m}, 7 \mathrm{H}, \mathrm{H}_{\mathrm{Ar}}\right) ; 6.68\left(\mathrm{~d}, J=8.9 \mathrm{~Hz}, 1 \mathrm{H}, \mathrm{H}_{\mathrm{Ar}}\right) ; 3.92\left(\mathrm{~s}, 2 \mathrm{H}, \mathrm{CH}_{2}\right)$. ${ }^{13}$ C NMR: 152.24 (s), 139.02 (s), 133.02 (s), 130.47 (d), 129.00 (s), 128.69 (d), 128.66 (d), 127.43 (d), 126.53 (d), 116.60 (d), 36.04 (t). - MS (70eV): $m / z$ (\%) 220 (34), 218 (100), 183 (34), 165 (19), 152 (21).

21: ${ }^{1} \mathrm{H}$ NMR: $7.41-7.28$ (m, 5H, $\mathrm{H}_{\mathrm{Ar}}$ ); 7.21/6.87 (AA'BB', $J=8.9 \mathrm{~Hz}, 2 \mathrm{H}, \mathrm{H}_{\mathrm{Ar}}$ ); 5.00 (s, $\left.2 \mathrm{H}, \mathrm{CH}_{2} \mathrm{O}\right) .-{ }^{13} \mathrm{C}$ NMR $\left(75 \mathrm{MHz}, \mathrm{CDCl}_{3}\right):=157.30$ (s), 136.54 (s), 129.30 (d), 128.59 (d), 128.05 (d), 127.40 (d), 125.75 (s), 116.11 (d), 70.20 (t).

1. Perrin, D. D.; Armarego, W. L. F. Purification of Laboratory Chemicals, 3. Edition, Pergamon Press, 1988.

2. Still, W. C.; Kahn, M.; Mitra, A. J. Org. Chem. 1978, 43, 2923-2925. 